\title{
Cranium Bifidum Occultum
}

\author{
S Vedajallam ${ }^{1}$, A Chacko ${ }^{1}$, S Andronikou ${ }^{2}$, R Truter $^{3}$, G White $^{4}$ \\ 1. Department of Radiology, Steve Biko Academic Hospital, University of Pretoria \\ 2. Department of Radiology, Faculty of Health Sciences, University of the Witwatersrand \\ 3. Schnetler, Corbett and Partners Radiology Practice, South Africa \\ 4. Neurosurgeon, Private Practice, Cape Town, South Africa \\ Corresponding Author: \\ Dr A Chacko \\ Postal Address: \\ Department of Radiology \\ Level 5, Bridge E \\ Steve Biko Academic Hospital \\ Voortrekker Road \\ Gezina \\ Pretoria \\ 0002 \\ Tel: $\quad+27123542406$ \\ Fax: $\quad+27865095150$ \\ Email: anithchacko@gmail.com
}

An infant girl was referred for assessment and management of a large frontal skull defect. She was born, after an uneventful pregnancy, by caesarian section to a 36-year-old mother (G1P1). The procedure was performed electively for a breech presentation. Her antenatal ultrasound had shown no specific abnormalities. The attending pediatrician immediately noted a large bi-frontal (but predominantly left-sided) skull defect confluent with a large anterior fontanelle. The overlying scalp was normal. APGARS were normal and the clinical examination was otherwise unremarkable. Ultrasound confirmed that there was no hydrocephalus and no other intracranial abnormality.

At initial neurosurgical evaluation, at 7 weeks age, the child showed a large bony defect above the brow on the left and reaching to a large anterior fontanelle (Figures 1a \& $b$ ). The defect also extended onto the right of the midline. There was an asymmetric leftsided mild bulging of the underlying frontal lobe into the defect. Neurological examination and development were normal, however.

CT scanning in bony windows (Figures $2 \mathrm{a}-\mathrm{c}$ ) demonstrated a bone defect within the left frontal bone. The coronal suture was intact and not widened. MRI (Figures $3 a \mathrm{a} b$ ) confirmed the herniation of the meninges and left frontal lobe through the defect in the left frontal bone.

A delayed surgical repair was performed using a right parietal calvarial graft. 


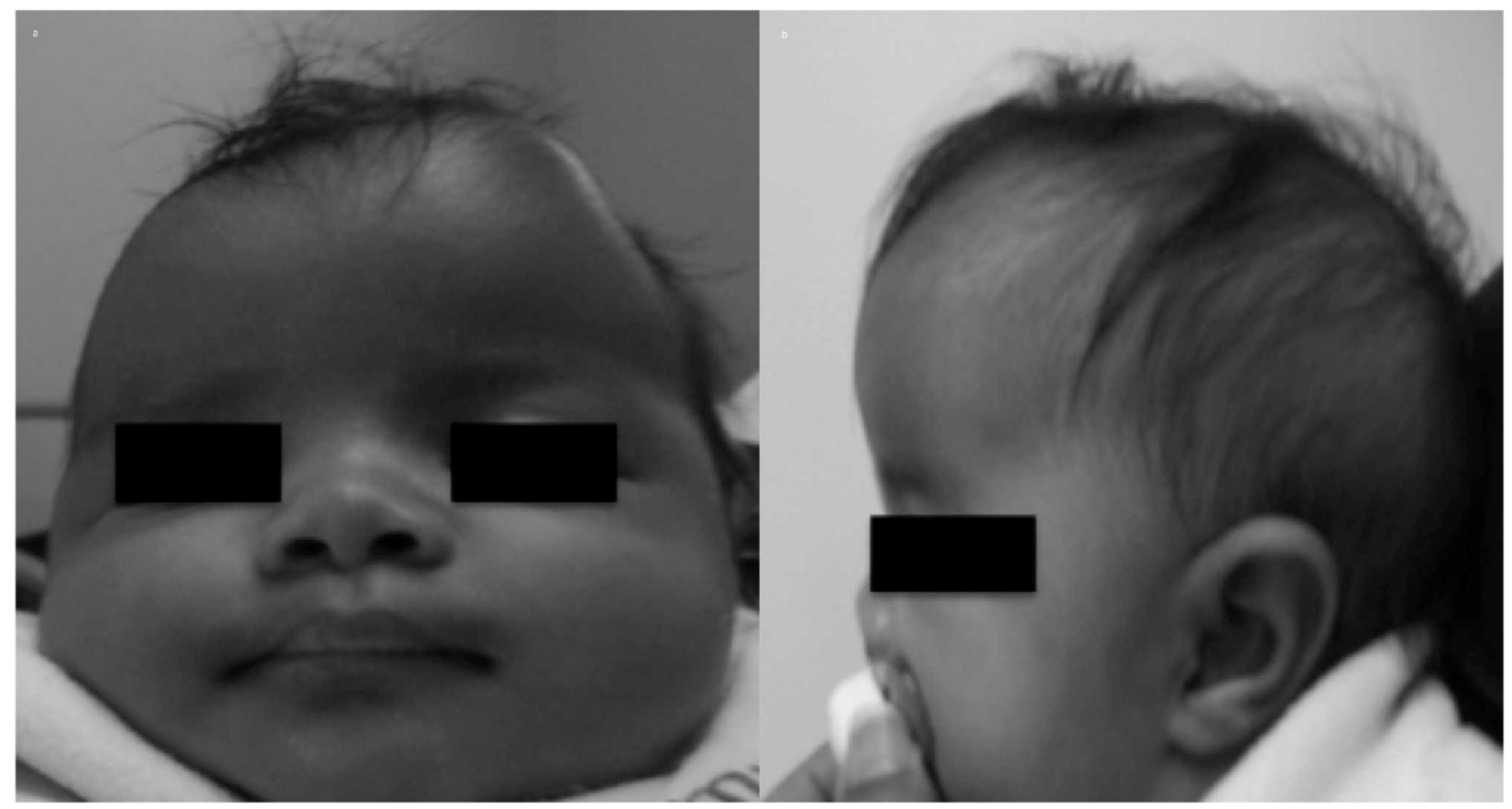

Figure 1 a-b: Clinical images obtained at initial neurosurgical consultation at the age of 7 weeks. Note the bulge over the left fronto-parietal region on the frontal (a) and lateral (b) photos.

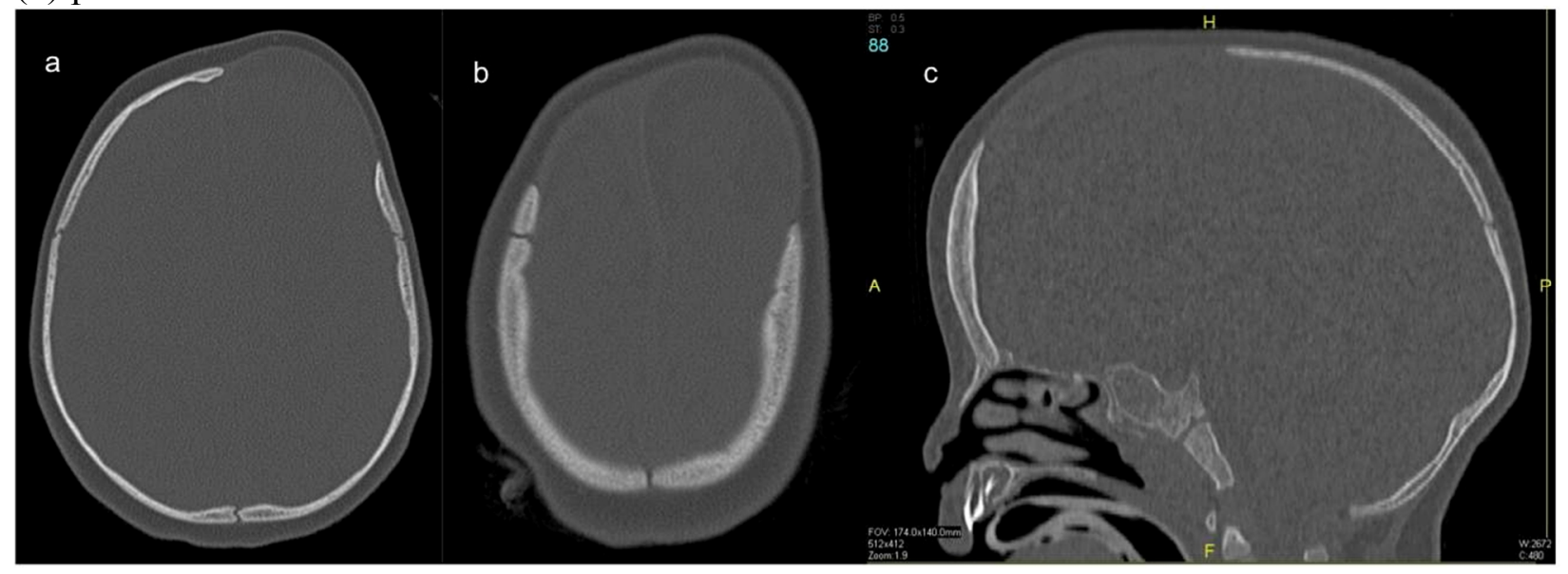

Figure 2 a-c: CT of the head utilizing 'bone windowing' - Axial image (a) demonstrates a large left frontal bone defect continuous with the anterior fontanelle on higher slices (b) with herniation of the intracranial contents. The coronal suture is intact and not widened. Parasagittal CT reconstruction of the skull to the left of midline (c) confirms the left frontal bone defect. 


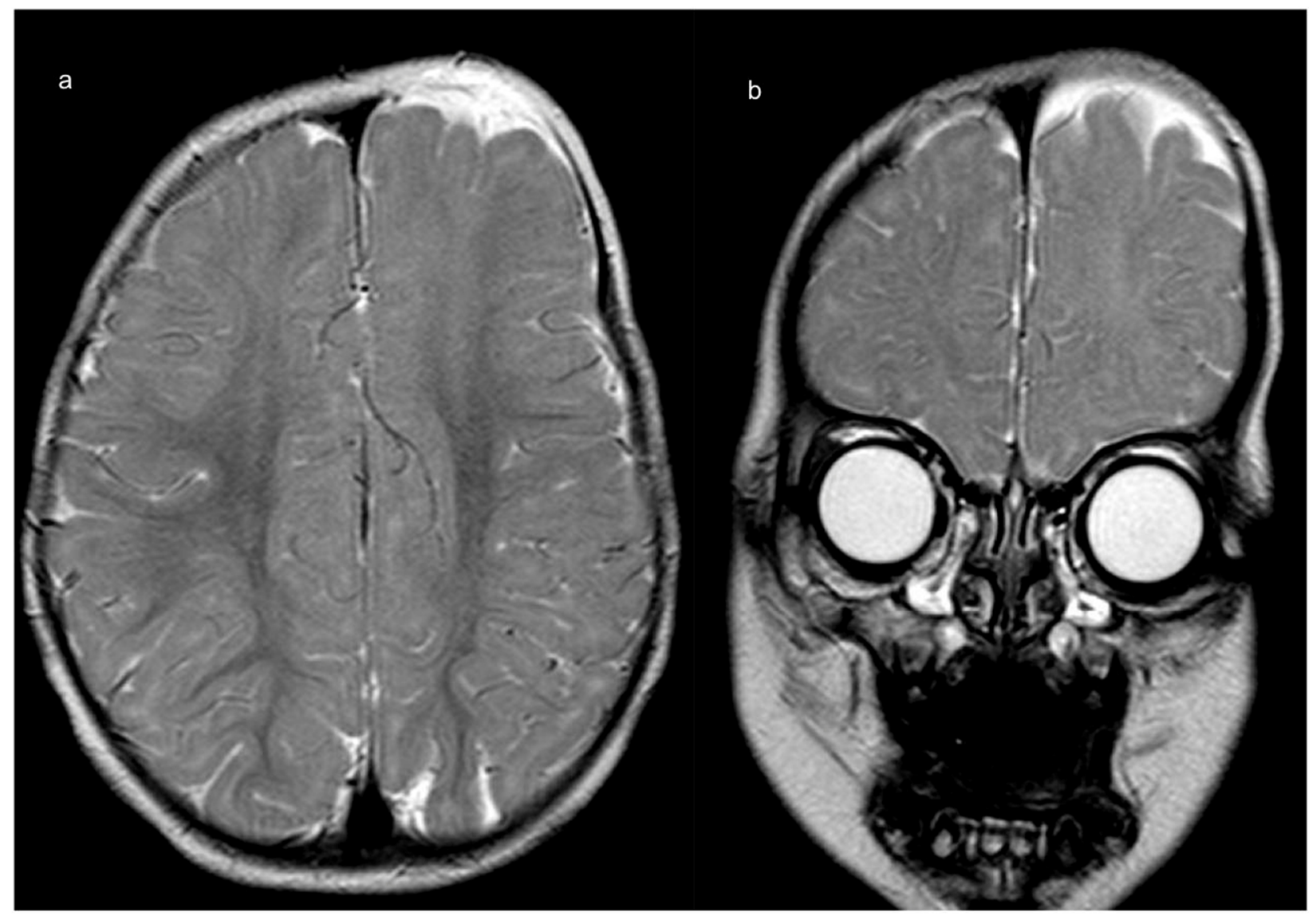

Figure 3 a and b: MRI of the brain - Axial (a) and coronal (b) T2-weighted images showing herniation of the meninges and left frontal lobe through the bone defect.

Cranium bifidum is an abnormal osseous defect of the calvarium through which herniation of meninges and brain tissue may occur. It occurs with mid-line malformations such as myelomeningocoele, meningoencephalocoele or dermal sinus. [1]

The appearance of cranium bifidum (also known as cleft skull or enlarged parietal foramina) is characterized by the unsuccessful midline migration of the cranial vault. [2] To our knowledge only five previously reported cases of true cranium bifidum exist in the literature.

During embryonal development the human skeleton undergoes two types of ossification. Long bones undergo mainly endochondral ossification whereas other bones (frontal, parietal and squamous parts of the temporal bones) undergo intra-membranous ossification. [3] During intra-membranous ossification the skull bones undergo direct ossification inside the vascularised membrane with the cranial bones growing and migrating towards the midline during development to completely cover the brain. [3]

True cranium bifidum, as in this patient, denotes an abnormal osseous defect through which there may be herniation of meninges and brain tissue of which only five cases have been reported previously. The formation of cranium bifidum may be the result of 
defective induction of the bone or failure of primary neural tube closure. [4,5] There are also often some scalp abnormalities associated with the clinical diagnosis. [5] Midline malformations such as myelomeningocoele, meningoencephalocoele, dermal sinus, or dural arteriovenous malformations [6] may occur but were not present in this patient. MRI is useful in identifying these.

Treatment of occult cranium bifidum is usually conservative due to the generally benign natural history. [7] In some cases persistent cranium bifidum may warrant surgical closure. [8] Any associated problems such as headaches or seizures are treated appropriately in a supportive manner. The risk of penetrating injury to the brain is present but small, however this may cause anxiety. Education of parents, teachers and later the affected child to avoid risky behavior that could result in such injury is necessary and usually sufficient. Contact sports should be avoided if the bony defect persists. [7]

True cranium bifidum is rare. Radiological recognition and assessment is important for diagnosis, detecting associations, planning of potential surgery and further management as well as further advising parents and the child, regarding lifestyle and sports.

\section{References}

1. Glass RBJ, Fernbach SK, Norton KI, et al. The infant skull: A vault of information. Radiographics 2004;24:507 - 522.

2. Celik SE \& Kara A. Complete cranium bifidum without scalp abnormality. J Neurosurg Pediatrics 2008;1:258 - 260.

3. Sadler TW. Langman's Medical Embryology 11th Ed. Wolters Kluwer and Lippincott, Williams \& Wilkins, 2004. Pgs 127 - 134.

4. Barkovitch AJ. Pediatric neuroimaging. 2nd ed. New York, NY: Raven, 1995.

5. Pendergrass EP \& Pepper OH. Observations on the process of ossification in the formation of persistent enlarged parietal foramina. Am J Roentgenol 1939;41:343-346.

6. Van Laak D \& Petridis AK. A case of occult cranium bifidum combined with abnormal high position of the straight sinus. Clinical Anatomy 2010;23: $87-878$.

7. Wilkie AOM \& Mavrogiannis LA. Enlarged parietal foramina/ Cranium bifidum. In: Pagon RA, Bird TD, Dolan TD, Stephens K, eds. GeneReviews [Internet]. Seattle (WA): University of Washington, Seattle; 1993 - 2004 Mar 30 [Updated 2010 Mar 30]. www.ncbi.nlm.nih.gov/books/NBK1128/ (Accessed April 2012)

8. Perlyn CA, Schmelzer R, Govier D, et al. Congenital scalp and calvarial deficiencies: principles for classification and surgical management. Plast Reconstr Surg 2005;115:1129-1149. 